\title{
Reversible and Efficient Light-Induced Molecular Switching on an Insulator Surface
}

Simon Jaekel, ${ }^{\dagger}$ Antje Richter, ${ }^{\ddagger}$ Robert Lindner, ${ }^{\ddagger}$ Ralf Bechstein, ${ }^{\ddagger}$ Christophe Nacci, ${ }^{\dagger}$ Stefan Hecht, ${ }^{\S}$ Angelika Kühnle, ${ }^{\ddagger}$ and Leonhard Grill*,†

${ }^{\dagger}$ Department of Physical Chemistry, University of Graz, Heinrichstrasse 28, Graz 8010, Austria

${ }^{\ddagger}$ Department of Physical Chemistry, University of Mainz, Duesbergweg 10-14, Mainz 55122, Germany

${ }^{\S}$ Department of Chemistry, Humboldt-Universität zu Berlin, Brook-Taylor-Straße 2, Berlin 10099, Germany

Supporting Information

ABSTRACT: Prototypical molecular switches such as azobenzenes exhibit two states, i.e., trans and cis, with different characteristic physical properties. In recent years various derivatives were investigated on metallic surfaces. However, bulk insulators as supporting substrate reveal important advantages since they allow electronic decoupling from the environment, which is key to control the switching properties. Here, we report on the light-induced isomerization of an azobenzene derivative on a bulk insulator surface, in this case calcite (1014), studied by atomic force microscopy with

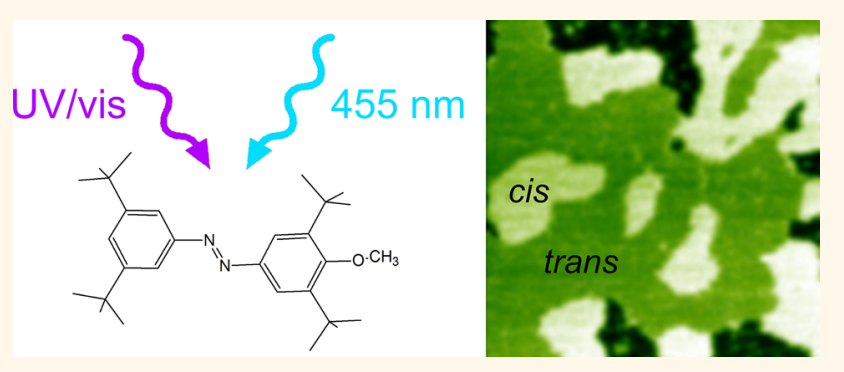
submolecular resolution. Surprisingly, cis isomers appear on the surface already directly after preparation, indicating kinetic trapping. The photoisomerization process is reversible, as the use of different light sources results in specific molecular assemblies of each isomer. The process turns out to be very efficient and even comparable to molecules in solution, which we assign to the rather weak molecular interaction with the insulator surface, in contrast to metals.

KEYWORDS: molecular switch, azobenzene, atomic force microscopy, bulk insulator, molecular assembly, photochemistry

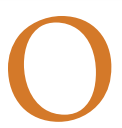

ver the past years, many studies have been devoted to designing, understanding, and exploiting molecular switches. ${ }^{1,2}$ Azobenzene is considered as a prototypical molecular switch with two conformational isomers, trans and cis, where the former is energetically preferred in gas phase and solution. It undergoes large structural changes during the trans $\leftrightarrow$ cis isomerization process, in contrast to tautomerization switches with more subtle changes that are advantageous if the packing and hence $2 \mathrm{D} / 3 \mathrm{D}$ assembly structure should be preserved. 3 The two isomeric, i.e., switching, states of azobenzene derivatives adsorbed on a surface can therefore be read-out rather easily by scanning probe microscopy. ${ }^{5-7}$ Accordingly, various azobenzenes have been studied with this method inducing isomerization with various stimuli ranging from tunneling electrons ${ }^{6-8}$ over electric fields in the molecule-tip junction ${ }^{5}$ to photons. 9,10

Beyond serving as a support to allow scanning probe microcopy to be used, the surface on which the molecular switches are adsorbed strongly influences the switching function itself. ${ }^{9}$ The stabilization of specific geometries by adsorption thus modulates potential barriers to be overcome during the switching process. Moreover, the interaction with the surface typically reduces the lifetimes of participating excited states, in particular on metallic substrates where photochemical transformations are frequently quenched. ${ }^{11}$
For this reason, it is important to decouple the azobenzene molecules from the metallic surface in order to ensure efficient isomerization. Most frequently, spacer groups have been used to lift the molecular core off metallic surfaces; however, the purely geometrical rationale of providing sufficient space for isomerization to occur is not sufficient to fully explain the observed switching behavior. ${ }^{12}$ It has been found that the isomerization of a given azobenzene derivative depends on the elemental composition as well as on the orientation of the surface. ${ }^{8}$ Furthermore, the precise adsorption site can allow or hinder isomerization of neighboring molecules within one close-packed molecular island. ${ }^{13}$ Metals are typically used for molecular adsorption, and metallic nanoparticles have for instance been functionalized with azobenzene derivatives to steer chemical reactions in solution. ${ }^{14}$ However, flat singlecrystal surfaces are the mostly used substrates for molecular switches, because they allow the use of scanning tunneling microscopy (STM) to directly image individual molecules before and after switching with superior spatial resolution. On the other hand, nonmetallic wide-gap substrates are attractive because they allow electronic decoupling of the molecules from

Received: December 5, 2017

Accepted: January 9, 2018

Published: January 9, 2018 
the surface, which is important for efficient switching processes and consequently better understanding. To the best of our knowledge, molecular photoswitches have not been investigated on an insulator surface thus far, despite their importance in miniaturized molecule-scale electronics. ${ }^{15-17}$

The investigation of molecular switches in real space is crucial because it allows correlating chemical processes in individual molecules with their local surroundings at the atomic scale. While STM, which also offers the possibility of singlemolecular manipulation via the tunneling electrons, ${ }^{18}$ is therefore the most popular method for metallic surfaces, studying molecular switches on insulators by scanning probe microscopy is challenging. First, atomic force microscopy (AFM) must be used instead of STM, rendering imaging more difficult when aiming for high resolution. ${ }^{19}$ This is due to the fact that the force does not typically decay exponentially with distance and that frequency modulation imaging requires controlling three interacting feedback loops compared to one in STM. Second, bulk insulators are often more difficult to prepare and/or exhibit more complicated unit cells than lowindex surfaces of noble metal crystals that are favored in STM studies. Finally, the interaction between the molecules and the supporting surface is typically weaker than between molecules and a metal surface. While this favors electronic decoupling, at the same time it commonly lowers the diffusion barrier or even leads to desorption, thus explaining the challenges encountered when aiming for imaging on insulator surfaces. ${ }^{20}$

Azobenzene derivatives have been investigated on ultrathin insulating $\mathrm{NaCl} \mathrm{films,}{ }^{21}$ where isomerization was not induced by light, but voltage/current pulses from the STM tip as the insulating layers are thin enough to allow electron tunneling. ${ }^{22}$ However, azobenzene switches have not been studied on bulk insulators so far. Here, we report the controlled reversible isomerization of azobenzene molecules on an insulating calcite surface using different light sources as analyzed by atomic force microscopy with submolecular resolution.

\section{RESULTS AND DISCUSSION}

Molecular Adsorption. As molecules we have chosen 4methoxy-3,3',5,5'-tetra-tert-butylazobenzene (M-TBA; Figure 1a) molecules, which have previously been studied by STM experiments ${ }^{13}$ and atomistic simulations ${ }^{23}$ upon adsorption on a $\mathrm{Au}(111)$ surface. In this study, the role of the symmetrically positioned bulky tert-butyl groups is primarily to facilitate the structural analysis by STM/AFM imaging. In cyclohexane solution, M-TBA exhibits characteristic azobenzene absorption spectra dominated by absorption bands at around $340 \mathrm{~nm}$ for the trans and at around $440 \mathrm{~nm}$ for the cis isomer, ${ }^{13}$ corresponding to $\pi \rightarrow \pi^{*}$ and $\mathrm{n} \rightarrow \pi^{*}$ excitations, respectively. M-TBA was deposited onto a calcite $\left(\mathrm{CaCO}_{3}\right)(10 \overline{1} 4)$ surface (Figure $1 \mathrm{~b}$ ), which not only is an important material in biomineralization $^{24}$ but also has been proven to provide an ideal substrate for studying the adsorption of organic molecules due to its ease of preparation and comparatively high surface energy of $590 \mathrm{~mJ} / \mathrm{m}^{2}$. $^{20,25}$

After deposition of the M-TBA molecules, three different regions can be distinguished in the AFM images as visible on the terrace in the upper right part of Figure 1c (a step edge is present at the lower left of the image). The three regions differ in apparent height: The area appearing black in Figure 1c corresponds to the bare calcite surface with some small bright protrusions that we ascribe to contaminations. In addition, islands of lower (dark in the image) and larger apparent height
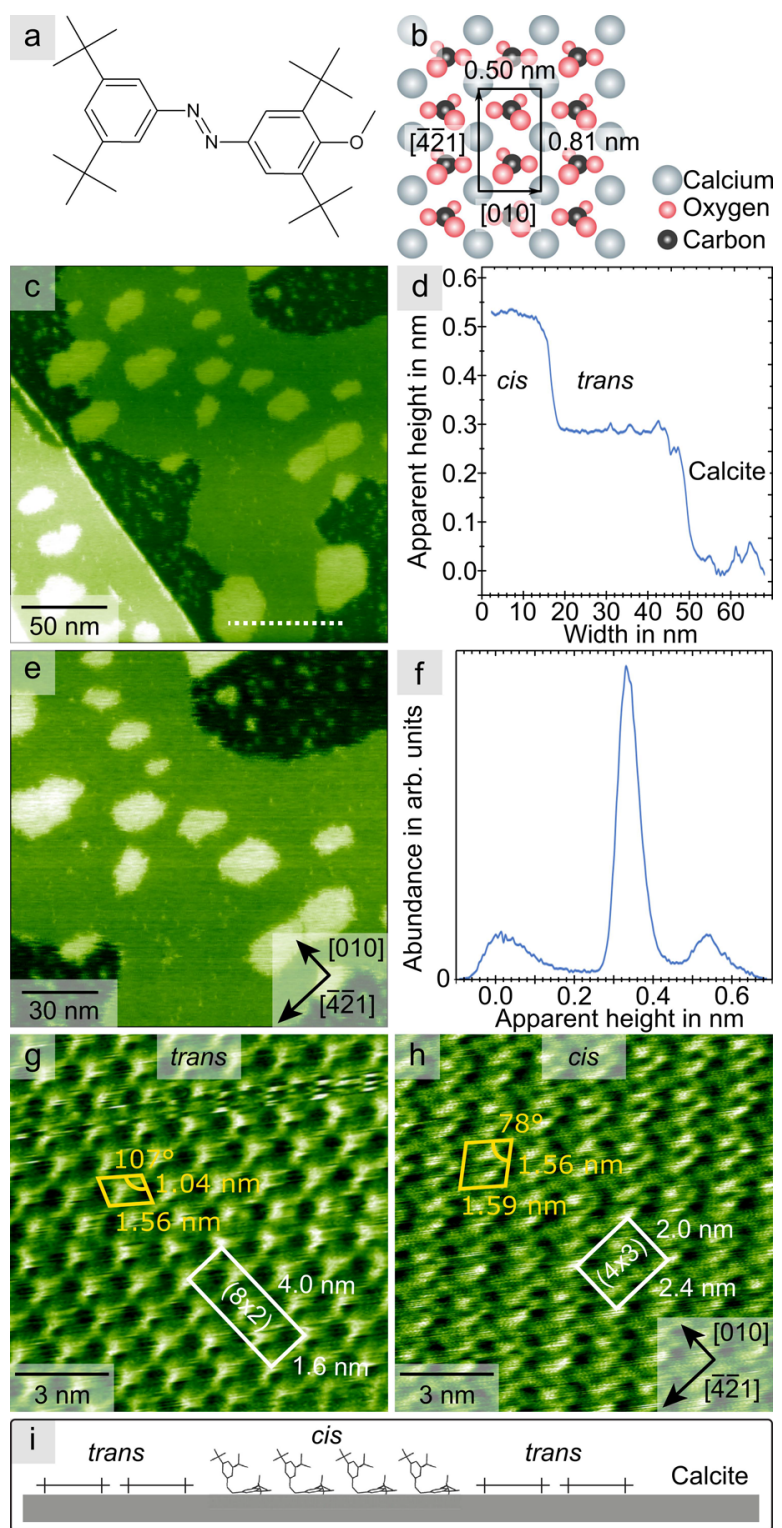

Figure 1. (a) Chemical structure of 4-methoxy-3,3',5,5' -tetra-tertbutylazobenzene (M-TBA) molecules. (b) Calcite $\left(\mathrm{CaCO}_{3}\right)(10 \overline{1} 4)$ surface. (c) AFM image after deposition of M-TBA onto the calcite surface with zoom-in in (e). (d) Height profile taken along the dashed line in (c). (f) Height distribution histogram of the AFM image in (e). AFM images of the lower molecular islands, assigned to trans isomers $(\mathrm{g})$ and the higher molecular islands $(\mathrm{h})$, assigned to $c i s$ isomers, on the surface (drawings show the primitive unit cells in yellow and the superstructure with respect to the calcite lattice in white). (i) Schematic view of the proposed structure with low trans and high cis islands.

(bright) are observed. The higher islands are often embedded in the lower ones (Figure 1c). The characteristic heights of these islands are $3.2 \pm 0.2$ and $5.2 \pm 0.3 \AA$, respectively (Figure le,f). We conclude from these values that the lower islands represent molecular monolayers of flat-lying M-TBA molecules in their trans configuration, because their apparent height fits to the size of a tert-butyl side group (molecular distances of $2.5 \AA$ for $\mathrm{C}$ and $4.1 \AA$ for $\mathrm{H}$ atoms within one tert-butyl group were determined from the single-crystal X-ray structure (see Supporting Information). Our interpretation is in agreement with manipulation experiments where individual molecules 
were removed from such islands and no structures with intermediate heights are found afterward. Instead, only the pure calcite surface was visible on the previous position of the molecule (see Supporting Information). We exclude cis isomers in this monolayer because these are characterized by a larger diameter (about at least $6 \AA$ in the gas phase), due to their more three-dimensional structure as compared to the rather flat trans isomer.

Concerning the brighter islands in the images, one possible interpretation is that they represent the second molecular layer that has grown on top of the first layer. However, a closer look into the lattice structures of the two areas shows that, while both structures are highly ordered in crystalline lattices, their lattices differ substantially. The unit cell of the lower structure has side lengths of $1.04 \pm 0.04 \mathrm{~nm}$ and $1.56 \pm 0.03 \mathrm{~nm}$ with an opening angle of $106.9 \pm 0.5^{\circ}$, while the higher structure exhibits $1.56 \pm 0.01 \mathrm{~nm}$ and $1.59 \pm 0.03 \mathrm{~nm}$ with an opening angle of $78 \pm 2^{\circ}$ (Figure 1g,h). In addition to these primitive unit cells, which are not aligned with the major substrate directions, we can assign larger unit cells (3-times for the lower (g) and 2-times for the higher structure (h)) that are aligned with the surface symmetry directions and reflect the commensurability with the calcite surface: $(8 \times 2)$ and $(4 \times 3)$ superstructures, respectively (Figure $1 \mathrm{~g}, \mathrm{~h}$ ).

AFM images of the lower layer (Figure $1 \mathrm{~g}$ ) reveal a regular pattern of bright protrusions. Intramolecular details are difficult in their interpretation since they change with the set-point of the $\Delta f$ feedback loop (see Supporting Information), while the position, size, and apparent height of the main protrusions remain, supporting our assignment of the unit cells. From the high-resolution AFM images, a unit cell area of $1.55 \pm 0.07 \mathrm{~nm}^{2}$ of this trans layer is determined. As each unit cell seems to contain one molecule, this area corresponds to a molecular density of $0.64 \pm 0.03$ molecules $/ \mathrm{nm}^{2}$, which is comparable to the density of trans isomers on $\mathrm{Au}(111)$ (between 0.59 and 0.75 molecules $\left./ \mathrm{nm}^{2}\right) .{ }^{13}$ We therefore assign each protrusion to an individual molecule, which is also in agreement with the distance between the protrusions (10.4 and $15.6 \AA$ ) in comparison with the molecular dimensions of about $9 \AA \times$ $15 \AA$ for trans M-TBA (determined from X-ray diffraction in the solid state, see Supporting Information).

The higher islands, which do not correspond to a second molecular layer, exhibit much larger unit cells $(2.43 \pm 0.05$ $\mathrm{nm}^{2}$ ) than the lower islands with flat-laying trans molecules $\left(1.55 \pm 0.07 \mathrm{~nm}^{2}\right)$, indicating the presence of more than one molecule per unit cell. We tentatively assign the molecules in the higher islands to cis isomers, twisted upward instead of lying flat (as sketched in Figure 1i), because the cis isomer exhibits a three-dimensional shape in the gas phase (as compared to the rather flat trans isomer), ${ }^{26}$ which is known to cause larger apparent heights upon adsorption on metal surfaces. ${ }^{5-8,10}$ Each unit cell seems to contain two cis molecules, corresponding to a molecular density of $0.82 \pm 0.02$ molecules $/ \mathrm{nm}^{2}$. An alternative assignment of three cis molecules per unit cell, giving rise to a molecular density of $1.23 \pm 0.03$ molecules $/ \mathrm{nm}^{2}$, appears less likely, as it would require a rather tightly packed structure with largely reduced molecular footprint, which has not been observed for M-TBA on metal surfaces. ${ }^{13}$ We want, however, to stress that a precise determination of the molecular density is difficult.

UV/Vis Illumination. In order to test whether our assignment of the higher islands to cis molecules is correct, we illuminated the sample using a broadband UV/vis lamp emitting between 300 and $600 \mathrm{~nm}$ (see Supporting Information). Indeed, with increasing illumination time (Figure $2 a-d)$ we see a decreased coverage of lower islands in favor of
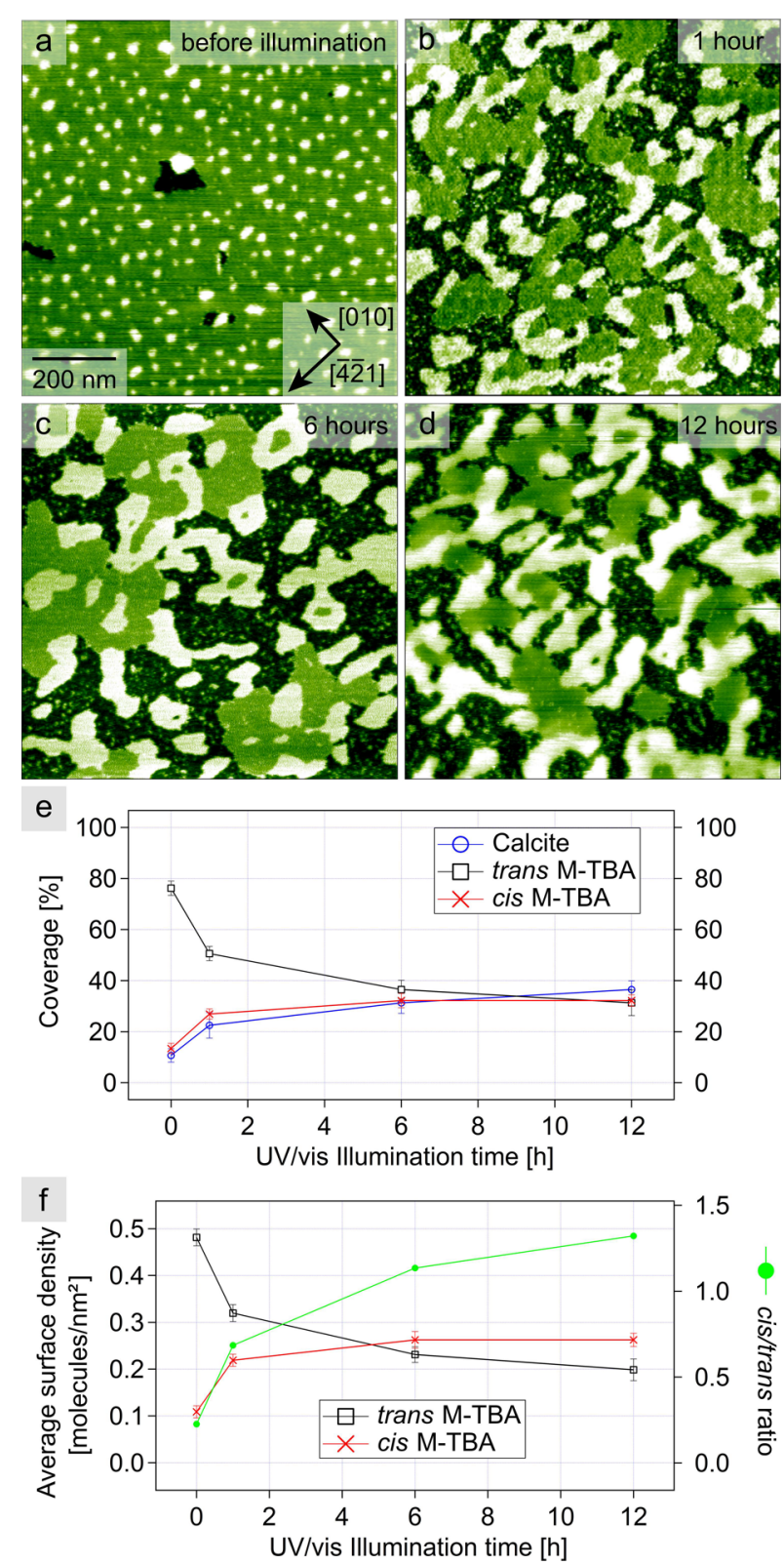

Figure 2. (a-d) AFM images (all $1 \times 1 \mu \mathrm{m}^{2}$ in size). (a) Directly after sample preparation and after illumination with a broadband $\mathrm{UV} /$ vis lamp (spectrum in the Supporting Information) for (b) $1 \mathrm{~h}$, (c) $6 \mathrm{~h}$, and (d) $12 \mathrm{~h}$. Development of the (e) surface coverage and (f) the surface density and cis/trans ratio of the molecules, plotted as a function of the illumination time (lines connect the data points to guide the eye). Statistics in (e) and (f) were obtained from surface areas of about $10 \mu \mathrm{m}^{2}$ for each data point.

the higher ones, in agreement with the photochromic character of the molecules (i.e., trans $\rightarrow$ cis isomerization). Note that the light absorption of the azobenzene molecules is much stronger in the UV range (allowed $\pi \rightarrow \pi^{*}$ transition) as compared to visible light (forbidden $\mathrm{n} \rightarrow \pi^{*}$ transition), resulting in a preference of trans $\rightarrow$ cis isomerization over the opposite direction (as the quantum yields in both isomerization directions do not differ significantly). ${ }^{27}$ Furthermore, the 

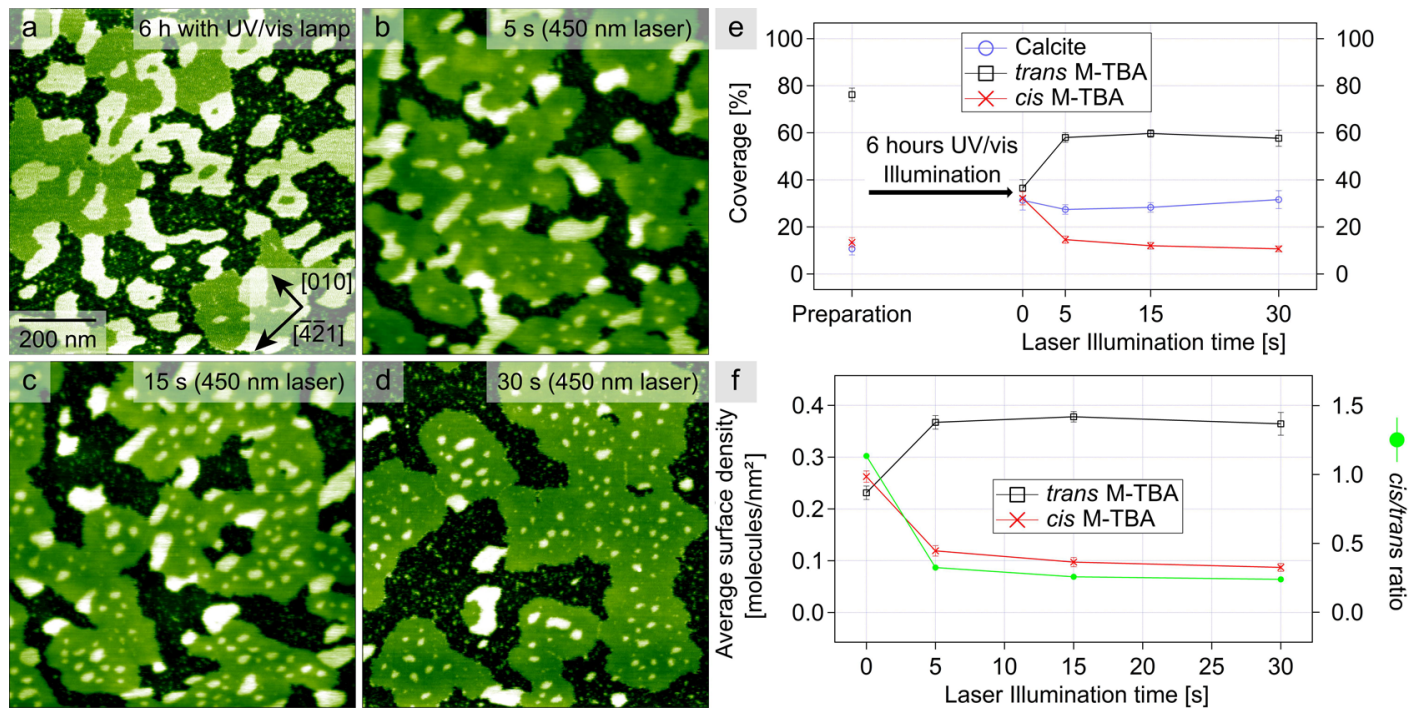

Figure 3. (a-d) AFM images (all $1 \times 1 \mu \mathrm{m}^{2}$ in size). (a) After molecular deposition and subsequent UV/vis lamp illumination for $6 \mathrm{~h}$, equivalent to Figure $2 \mathrm{c}$. AFM images after illumination with a $450 \mathrm{~nm}$ laser (details in the Supporting Information) for (b) $5 \mathrm{~s}$, (c) $15 \mathrm{~s}$, and (d) $30 \mathrm{~s}$. (e, f) Temporal evolution of the relative surface coverages and the surface densities of the different areas (calcite and trans and cis islands). The cis/trans ratio of the molecules is plotted on the right axis of (f). Assuming a simple first-order desorption process, the experimental curves were fitted with a single-exponential function. Statistics in (e) and (f) were obtained from surface areas of about $10 \mu \mathrm{m}^{2}$ for each data point.

trans isomer is typically thermodynamically more stable and therefore expected to be dominant directly after preparation and before illumination, which is the case (Figure $2 \mathrm{a}$ ). Hence, the initial surface (Figure 2a) is dominated by large areas of trans monolayer islands that contain many small cis islands.

Our data show that adsorption of M-TBA on calcite differs from that on metallic surfaces. On the latter, M-TBA is found in the trans state only, ${ }^{13}$ and the same is valid for other isomerizing switches. ${ }^{5-7,10}$ An exception has been found for imine derivatives that were found to spontaneously switch from trans to cis with increasing coverage, driven by the reduced surface required by the cis isomers. ${ }^{28}$ The characteristic preference of the trans state on metals can be understood from a thermodynamic point of view. While cis M-TBA exhibits a half-life of $330 \mathrm{~h}$ at $30{ }^{\circ} \mathrm{C}$ in solution, ${ }^{13}$ it experiences temperatures around $90{ }^{\circ} \mathrm{C}$ during sublimation. It is likely that both isomers (majority of trans with minor amounts of cis) arrive at the surface during preparation, but only the energetically favored trans species survives, because a warm metallic surface (kept at room temperature during deposition) enables thermal cis $\rightarrow$ trans isomerization. In order to test this hypothesis, we have deposited M-TBA molecules onto a cold $\mathrm{Au}(111)$ surface (kept at $10 \mathrm{~K}$ ) and indeed found also cis isomers on the surface (see Supporting Information). This shows for the metallic surface that cis isomers adsorb from the gas phase, but cannot relax to the trans state after adsorption at these temperatures, due to the lack of thermal energy, and therefore do not reach their thermodynamic minimum.

In contrast, on the calcite surface cis isomers of M-TBA are found even if the sample is kept at room temperature (e.g., Figure 1c). They thus seem to be kinetically trapped at the calcite surface after adsorption. Note that M-TBA molecules only diffuse on the calcite surface at room temperature, but do not isomerize spontaneously (as discussed in detail below). Accordingly, the cis isomers on the calcite surface seem to be not created on the surface, but reflect the adsorption from the gas phase, in agreement with the interpretation above.
Interestingly, the cis isomers prefer to form (many) small islands rather than (a few) larger ones on the calcite surface, although the latter could be expected if island growth is energydriven, as close-packing would be more efficient for larger ordered arrays. The opposite preference here might be caused by a limited molecular diffusion, i.e., substantial adsorbatesurface interaction and nucleation-dominated growth, of cis isomers that limits the island size. Such an effect could be enhanced by calcite defects that kinetically trap molecules and act as nucleation centers for the cis islands, which are therefore small in size, but large in number.

Photoswitching of the M-TBA molecules can be quantified by measuring the relative surface area of the three different components - calcite and trans and cis isomers-after different illumination times (Figure 2e). Note that the relative coverages are directly proportional to the absolute coverages, because the three analyzed structures represent all existing species and thus the entire surface. It can be clearly seen how the relative areas of calcite and cis islands increase, while that of the trans islands decreases with the illumination time. The spectrum of our UV/ vis lamp covers both absorption bands of the M-TBA molecules in solution, at about $330 \mathrm{~nm}$ (trans $\rightarrow$ cis) and $440 \mathrm{~nm}$ (cis $\rightarrow$ trans) ${ }^{13}$ thus inducing both isomerization processes (trans $\rightarrow$ cis and cis $\rightarrow$ trans). However, we start with a rather small cis coverage (as compared to trans), and therefore trans $\rightarrow$ cis isomerization prevails at the beginning.

In order to identify the photostationary state (PSS), we do not plot the relative coverages, but the absolute number of molecules per surface area (Figure 2f), calculated by using molecular packing densities determined from the unit cells (Figure 1g,h). From these we derive the cis/trans ratio (green dots in Figure 2f), which continuously increases, even after 12 h. Hence, the PSS is not reached during our observation times, but rather after approximately $15 \mathrm{~h}$, where a constant cis/trans ratio is reached in extrapolation. We also find that, in addition to the reversible trans $\leftrightarrow$ cis isomerization, molecular desorption, caused by the photoexcitation, ${ }^{29}$ affects the (trans, 
cis, and calcite) coverages and causes a decrease of the total number of molecules per surface area (see Supporting Information). This explains why the surface densities remain constant only for cis isomers but continue to decrease for trans molecules for longer illumination times (Figure 2f). If we assume that both isomers desorb upon illumination, then the total number of cis isomers would decrease after about 6-12 h.

However, at the same time the system is approaching its photostationary state, thus continuously increasing the relative number of cis isomers (i.e., the trans $\rightarrow$ cis isomerization prevails over cis $\rightarrow$ trans), and these two effects compensate each other, resulting in an approximately constant cis surface density (Figure 2f). It should be mentioned that we cannot access the individual desorption rates of the two isomers, because the isomerization occurs faster than desorption (Figures S4 and S5). Hence, after each illumination step the system equilibrates into the photostationary state, and consequently both surface coverages (trans and cis) are reduced in our images to keep the cis/trans ratio constant, although maybe only one of the two isomers is photodesorbed. Note that no temperature increase was measured on the sample holder (illuminated together with the sample) during any of these experiments. Hence, photon heating appears unlikely to cause either isomerization or desorption of the molecules.

Reversibility of Photoisomerization. An important property of azobenzene derivatives and arguably any other useful photoswitch is the reversibility of the molecular interconversion and associated reversible property changes, whereas irreversible isomerization processes ${ }^{30}$ are barely of interest for the use of molecular switches. Hence, the question arises whether the trans $\rightarrow$ cis switching obtained with a UV/vis lamp can be reversed by using another light source with appropriate wavelength. Since the dominant cis $\rightarrow$ trans absorption band of M-TBA in solution is located at about $440 \mathrm{~nm},{ }^{13}$ we have chosen a $450 \mathrm{~nm}$ laser. A series of AFM images (Figure $3 \mathrm{a}-\mathrm{d}$ ) reveals that the bright islands (cis) are reduced in size in favor of the dark islands (trans). Hence, this specific wavelength can preferentially isomerize from cis to trans (in contrast to the UV/vis lamp before). It is evident from the AFM images that the calcite surface areas are more abundant after a complete cycle, a result of molecular desorption as the total number of molecules on the surface decreases also for laser illumination (see Supporting Information). It is clear that the initial surface morphology (i.e., directly after molecular deposition and before illumination; Figure 2a) is found again (Figure 3d). This indicates substantial molecular mobility of the trans isomers, which (e.g., after cis $\rightarrow$ trans isomerization) sufficiently diffuse on the surface to engulf the small cis islands. At the same time, the cis isomers seem to be immobilized and the characteristic pattern of small bright cis islands within large dark trans areas is recovered, supporting the interpretation of calcite defects kinetically trapping cis isomers (see above). Hence, the entire isomerization cycle trans (Figure 2a) $\rightarrow$ cis (Figures $2 \mathrm{c}$ and $3 \mathrm{a}$ ) $\rightarrow$ trans (Figure $3 \mathrm{~d}$ ) is indeed reversible.

Note that more and more of the calcite surface (black) becomes visible upon illumination, which we assign to two processes: (i) conversion from a less into a more densely packed structure (i.e., trans $\rightarrow$ cis) upon illumination with the $\mathrm{UV} /$ vis lamp (Figure $2 \mathrm{a}-\mathrm{d}$ ) and (ii) molecular desorption. The importance of the latter becomes particularly evident when studying the surface areas of free calcite after a complete trans $\rightarrow$ cis $\rightarrow$ trans cycle (i.e., comparing Figure $3 d$ with Figure 2a).
Photoisomerization Efficiency. An important piece of information obtained from these experiments is the short time scale on which switching occurs (seconds as compared to hours in Figure 2), which becomes even more evident when plotting the time evolution of the surface composition (Figure 3e,f). It is clear not only that the isomerization is reversed as compared to $\mathrm{UV} /$ vis lamp illumination, as the cis/trans ratio decreases (Figure 3f), but also how quickly this takes place. The relative calcite surface area clearly shrinks within the first $5 \mathrm{~s}$ of laser illumination, because the trans isomers simply cover more surface area per molecule (Figure 1). However, it starts to increase for longer illumination times beyond $5 \mathrm{~s}$ (Figure 3e), indicating molecular desorption (see Supporting Information). The same is found when comparing the calcite areas in the initial and final AFM images of the entire sequence (Figures $2 \mathrm{a}$ and $3 \mathrm{~d})$.

By fitting the data points with exponential functions, we obtain decay constants of about 2-3 s for the evolution of trans and cis areas and thus a very fast process as compared to other studies: An extremely low conversion rate has been obtained for the azobenzene derivatives TBA on a $\mathrm{Au}(111)$ surface where the stationary state is obtained only after light exposure to $\sim 5 \times 10^{22}$ photons $/ \mathrm{cm}^{2}$, ${ }^{10}$ which corresponds to about $80 \mathrm{~h}$ of illumination with $375 \mathrm{~nm}$ (despite a power density of 92 $\mathrm{mW} / \mathrm{cm}^{2}$ as compared to $24 \mathrm{~mW} / \mathrm{cm}^{2}$ in the present study). This inefficient process can be understood from the photon energy since the $375 \mathrm{~nm}$ light source does not match any absorption band for photoisomerization (known from solution experiments). ${ }^{31}$ However, even if a more appropriate wavelength $(300 \mathrm{~nm})$ is used, it takes 5 to $10 \mathrm{~min}$ to reach the PSS, despite the very high power density of $16.9 \mathrm{~W} / \mathrm{cm}^{2} .^{31}$ This is 1 to 2 orders of magnitude slower than in the present case with only $24 \mathrm{~mW} / \mathrm{cm}^{2}$.

In order to give a more quantitative comparison, we have calculated the cross section of the photoisomerization in our case and found $\Phi_{\text {trans } \rightarrow \text { cis }}=1.4 \times 10^{-18} \mathrm{~cm}^{2}$ and $\Phi_{\text {cis } \rightarrow \text { trans }}=5.9 \times$ $10^{-18} \mathrm{~cm}^{2}$ for illumination with the $455 \mathrm{~nm}$ laser. Other cross sections reported for azobenzene derivatives adsorbed on metal surfaces are $6 \times 10^{-20} \mathrm{~cm}^{2}$ at a broad spectrum of wavelengths between 330 and $600 \mathrm{~nm},,^{32} 4 \times 10^{-21} \mathrm{~cm}^{2}$ at $282 \mathrm{~nm},{ }^{31} 8 \times$ $10^{-22} \mathrm{~cm}^{2}$ at $300 \mathrm{~nm},{ }^{31}$ about $2 \times 10^{-23} \mathrm{~cm}^{2}$ at 375 and 444 $\mathrm{nm},{ }^{10}$ and $2.1 \times 10^{-24} \mathrm{~cm}^{2}$ at $318 \mathrm{~nm} .{ }^{33}$ Hence, isomerization occurs very quickly in the present case, as the values obtained with the $450 \mathrm{~nm}$ laser are at least 2 orders of magnitude larger than experimental values on metals. Note that cross sections for our illumination with the UV/vis lamp $\left(\Phi_{\text {trans } \rightarrow \text { cis }}=3.5 \times 10^{-20}\right.$ $\mathrm{cm}^{2}$ and $\Phi_{\text {cis } \rightarrow \text { trans }}=2.9 \times 10^{-20} \mathrm{~cm}^{2}$ ) are smaller than for the $455 \mathrm{~nm}$ laser, probably due to less suitable matching with the absorption bands, but still rather large as compared to previous studies. This pronounced improvement in terms of switching efficiency in the present case can be attributed to the use of an insulator surface, instead of a metal. Accordingly, the molecules adsorb very weakly and the typical effects for reduced isomerization efficiency on a metallic surface are absent: (1) direct interaction with the metallic surface, leading to metalorganic bonding that reduces the molecular flexibility required during the conformational changes; ${ }^{34}$ (2) hybridization with the metallic surface that causes either quenching of excited states that are involved in the isomerization process ${ }^{35}$ or (3) direct changes of the optical absorption properties. ${ }^{9}$ Note that our cross sections are comparable to those for azobenzene derivative trans $\rightarrow$ cis isomerization in solution $\left(\sim 10^{-19} \mathrm{~cm}^{2}\right)^{9}$ and thus illustrate that the use of an insulator surface indeed 
preserves the intrinsic photochromic properties of the molecules.

Development of Surface Morphologies. The surface morphology turns out to be not only characteristic for each stage during the isomerization cycle but also very reversible. We have studied this by following the evolution of islands with different size during the entire trans $\rightarrow$ cis $\rightarrow$ trans sequence (Figure 4). While almost exclusively very small cis islands (i.e.,

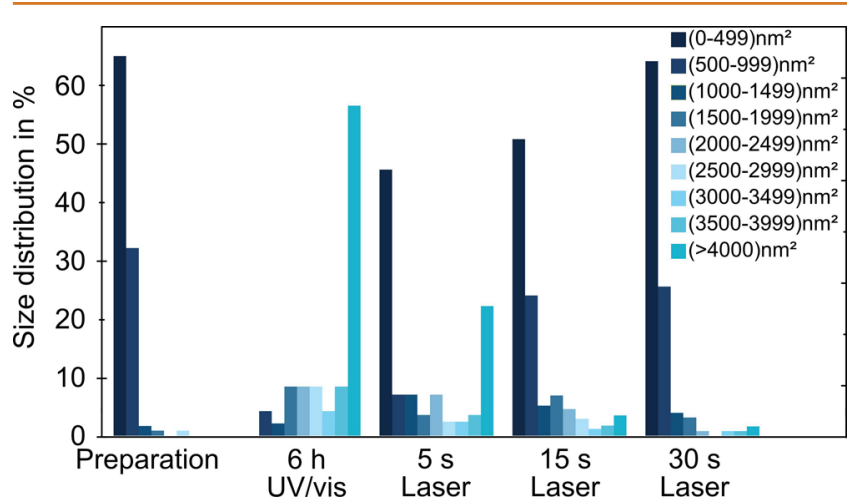

Figure 4. Size distribution of cis islands on the surface for different stages during an entire trans $\rightarrow$ cis $\rightarrow$ trans cycle, starting with the original surface directly after sublimation (labeled "Preparation"), then illumination with the UV/vis lamp (" $6 \mathrm{~h} \mathrm{UV} /$ vis") first and the $450 \mathrm{~nm}$ laser (for 5, 15, and $30 \mathrm{~s}$, respectively) afterward.

areas below $1000 \mathrm{~nm}^{2}$ ) are present directly after sample preparation, these islands increase in size upon $6 \mathrm{~h} \mathrm{UV} / \mathrm{vis}$ lamp illumination, and very small islands $\left(<500 \mathrm{~nm}^{2}\right)$, initially the dominant feature, completely disappear. Instead, very large islands $\left(4000 \mathrm{~nm}^{2}\right)$ emerge as the by far most abundant species. Importantly, the small islands are well restored upon illumination with the $450 \mathrm{~nm}$ laser, causing a continuous increase of the islands' size with illumination time. Finally, after completing the entire switching cycle, almost exactly the same surface morphology is obtained as in the initial state (histograms on the left and right edge in Figure 4).

Molecular diffusion can of course play a role in these processes since trans and cis isomers could in principle exhibit different diffusion barriers and therefore mobilities. We have therefore tested this property by following the evolution of one and the same surface area over several hours without any illumination, thus only allowing thermally driven processes at room temperature (Figure $5 \mathrm{a}-\mathrm{c}$ ). Note that under these conditions no isomerization takes place and total surface areas of trans and cis islands remain constant (not within a single image though where diffusion in/out of the scanned surface area occurs). However, the spatial distribution does not change significantly, as evident in AFM image sequences (Figure 5ac).

The surface area chosen for Figure 5 contains all surface structures of interest: pure calcite, trans islands (dark), and cis islands (bright) in both variations, surrounded by trans areas and in direct vicinity of calcite. From comparison of the two AFM images or the difference image (Figure 5d), it becomes clear that the trans molecules are mobile. They continuously detach from (or attach to) edges of the trans islands, which consequently change their shape (note that for the particular case in Figure $5 \mathrm{a}-\mathrm{c}$ the total number of molecules is reduced, as there is a net molecule diffusion out of the image area). In contrast to this, the cis islands that are embedded in trans
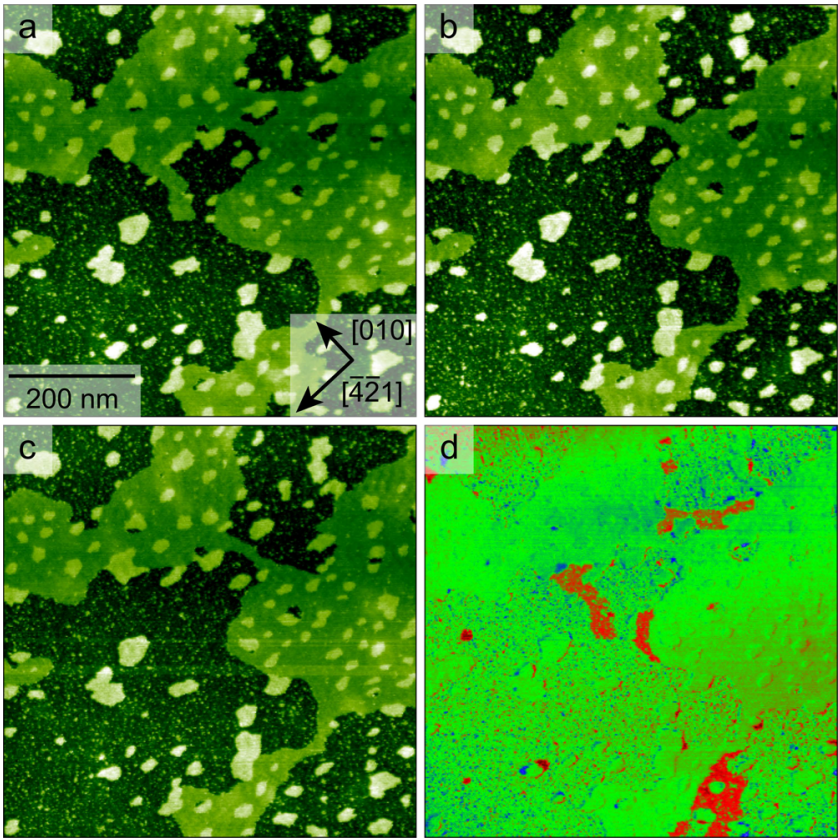

Figure 5. (a-c) AFM images, taken at room temperature, of exactly the same sample area imaged within $4 \mathrm{~h}$ ( $2 \mathrm{~h}$ between each image) without illumination. (d) Corresponding difference image between (a) and (c) to investigate the diffusion properties (with green being unchanged areas, red with reduced, and blue with enlarged apparent height in the image).

islands do not change at all; hence, this species is completely immobile. At the same time, the cis islands that are located next to calcite areas do change, although not very strongly. Altogether, this means that trans isomers are much more mobile on the calcite surface than cis isomers, which we attribute to a stronger molecule-calcite interaction in the case of the cis isomer, in agreement with the interpretation of limited diffusion of $c i s$ isomers above. The embedded cis isomers can instead not diffuse at all, simply because they are hindered by the trans molecules surrounding them.

Cooperative trans $\rightarrow$ cis Photoisomerization. An important qualitative observation is the almost complete absence of single cis isomers within the trans islands upon illumination with $450 \mathrm{~nm}$ (see Figure 2d), in contrast to azobenzene derivatives on metallic surfaces, where random switching of individual molecules occurs. ${ }^{5}$ This could be interpreted as an indication for cooperative switching where the switching state of one molecule influences its direct neighbor, ${ }^{36,37}$ because cis molecules cannot diffuse efficiently within the trans islands (Figure $5 \mathrm{a}-\mathrm{c}$ ). Consequently, individual cis isomers should be distributed randomly within the dark trans islands upon light-induced trans $\rightarrow$ cis isomerization, which is not the case; they are found in islands instead (Figure 2). We therefore conclude that the assembly of cis isomers in islands is most likely not caused by thermal diffusion, but might be rather the result of cooperative switching, where the trans $\rightarrow$ cis isomerization is more efficient in the presence of one or several cis isomers in the direct molecular surrounding (i.e., at the transition between trans and cis areas). Hence, illumination results in the growth of cis islands rather than the appearance of individual cis isomers in the trans islands. 


\section{CONCLUSIONS}

Our study gives experimental evidence for kinetic trapping of cis isomers on a calcite surface, in contrast to the gas phase or metallic surfaces, where exclusively trans isomers are found. Illumination allows selective switching between the two isomer states by applying suitable light sources and wavelengths. This process turns out to be reversible in terms of both the abundance of trans and cis isomers on the calcite surface as well as the size distribution of the islands. The cis isomers always appear in islands, which we attribute to a possible molecular cooperativity in the trans $\rightarrow$ cis switching.

Photoisomerization is found to be very efficient on the calcite surface, and very high cross sections are determined. They exceed the values previously determined on metals by at least 2 orders of magnitude and are instead comparable to those in solution. This observation proves that the insulating calcite surface does not interfere with the photoisomerization and instead preserves the intrinsic photochromic properties of the molecules.

\section{METHODS}

Experiments were performed under ultrahigh vacuum (UHV) with a base pressure of $10^{-10}$ mbar. Calcite crystals (purchased at Korth Kristalle) were prepared by first annealing at $250{ }^{\circ} \mathrm{C}$ for $3 \mathrm{~h}$, then in situ cleaving, and finally annealing at $200{ }^{\circ} \mathrm{C}$ for 45 to $90 \mathrm{~min}$. Molecules were deposited for 3 to $6 \mathrm{~min}$ from a Knudsen cell at a temperature of about $90{ }^{\circ} \mathrm{C}$, while keeping the sample at room temperature. AFM measurements were performed at room temperature with a VT-AFM (ScientaOmicron), operated in the frequency modulation mode. We used silicon cantilevers from Nanosensors with eigenfrequencies of about $300 \mathrm{kHz}$ in UHV and spring constants on the order of $40 \mathrm{~N} / \mathrm{m}$.

Illumination of the sample was done with (1) a UV/vis lamp emitting between 300 and $600 \mathrm{~nm}$ and a power density of $3.09 \mathrm{~mW} /$ $\mathrm{cm}^{2}$ through a (Silux) UHV window with $>90 \%$ transmission and (2) a $450 \mathrm{~nm}$ laser (spot size: about $19 \mathrm{~mm}^{2}$ with a total power of $4.5 \mathrm{~mW}$ ) through a borosilicate window with maximum transmission (about $90 \%$ ) between $400 \mathrm{~nm}$ and $2 \mu \mathrm{m}$ (see Supporting Information).

\section{ASSOCIATED CONTENT}

\section{S Supporting Information}

The Supporting Information is available free of charge on the ACS Publications website at DOI: 10.1021/acsnano.7b08624.

Details on AFM experiments and the illumination setup; deposition of M-TBA on the cold $\mathrm{Au}(111)$ surface; molecular geometry from X-ray crystal diffraction; isomerization cross sections (PDF)

\section{AUTHOR INFORMATION}

\section{Corresponding Author}

*E-mail: leonhard.grill@uni-graz.at.

\section{ORCID}

Stefan Hecht: 0000-0002-6124-0222

Angelika Kühnle: 0000-0003-1214-1006

Leonhard Grill: 0000-0002-9247-6502

\section{Author Contributions}

L.G. and A.K. conceived the experiments, S.J., R.L., and A.R. performed them. C.N. performed and analyzed the experiments on the $\mathrm{Au}(111)$ surface. S.J., A.K., and L.G. analyzed the data. S.H. provided the molecules and analyzed their switching in solution. The manuscript was written by L.G. through contributions of all authors.

\section{Notes}

The authors declare no competing financial interest.

\section{ACKNOWLEDGMENTS}

We thank the European Marie Curie Initial Training Network (ITN) for financial support through the ACRITAS project. Jutta Schwarz is acknowledged for help with the synthesis of MTBA and Bernd M. Schmidt for assistance with refining its Xray crystal structure.

\section{REFERENCES}

(1) Feringa, B. L.; Browne, W. R. Molecular Switches; Wiley-VCH: Weinheim, 2011.

(2) Molen, S. J. v. d.; Liljeroth, P. Charge Transport through Molecular Switches. J. Phys.: Condens. Matter 2010, 22, 133001.

(3) Liljeroth, P.; Repp, J.; Meyer, G. Current-Induced Hydrogen Tautomerization and Conductance Switching of Naphthalocyanine Molecules. Science 2007, 317, 1203-1206.

(4) Fita, P.; Grill, L.; Listkowski, A.; Piwonski, H.; Gawinkowski, S.; Pszona, M.; Sepiol, J.; Mengesha, E.; Kumagai, T.; Waluk, J. Spectroscopic and Microscopic Investigations of Tautomerization in Porphycenes: Condensed Phases, Supersonic Jets, and Single Molecule Studies. Phys. Chem. Chem. Phys. 2017, 19, 4921-4937.

(5) Alemani, M.; Peters, M. V.; Hecht, S.; Rieder, K.-H.; Moresco, F.; Grill, L. Electric Field-Induced Isomerization of Azobenzene by STM. J. Am. Chem. Soc. 2006, 128, 14446-14447.

(6) Henzl, J.; Mehlhorn, M.; Gawronski, H.; Rieder, K.-H.; Morgenstern, K. Reversible cis-trans Isomerization of a Single Azobenzene Molecule. Angew. Chem., Int. Ed. 2006, 45, 603-606.

(7) Choi, B.-Y.; Kahng, S.-J.; Kim, S.; Kim, H.; Kim, H. W.; Song, Y. J.; Ihm, J.; Kuk, Y. Conformational Molecular Switch of the Azobenzene Molecule: A Scanning Tunneling Microscopy Study. Phys. Rev. Lett. 2006, 96, 156106.

(8) Alemani, M.; Selvanathan, S.; Moresco, F.; Rieder, K.-H.; Ample, F.; Joachim, C.; Peters, M. V.; Hecht, S.; Grill, L. Adsorption and Switching Properties of Azobenzene Derivatives on Different Noble Metal Surfaces; $\mathrm{Au}(111), \mathrm{Cu}(111)$ and $\mathrm{Au}(100)$. J. Phys. Chem. C 2008, 112, 10509-10514.

(9) Tegeder, P. Optically and Thermally Induced Molecular Switching Processes at Metal Surfaces. J. Phys.: Condens. Matter 2012, 24, 394001.

(10) Comstock, M. J.; Levy, N.; Cho, J.; Berbil-Bautista, L.; Crommie, M. F.; Poulsen, D. A.; Frechet, J. M. J. Measuring Reversible Photomechanical Switching Rates for a Molecule at a Surface. Appl. Phys. Lett. 2008, 92, 123107.

(11) Hagen, S.; Kate, P.; Leyssner, F.; Nandi, D.; Wolf, M.; Tegeder, P. Excitation Mechanism in the Photoisomerization of a SurfaceBound Azobenzene Derivative: Role of the Metallic Substrate. J. Chem. Phys. 2008, 129, 164102

(12) McNellis, E. R.; Bronner, C.; Meyer, J.; Weinelt, M.; Tegeder, P.; Reuter, K. Azobenzene versus Tetra-Tert-Butyl-Azobenzene at $\mathrm{Au}(111)$ : Characterizing the Role of Spacer Groups. Phys. Chem. Chem. Phys. 2010, 12, 6404-6412.

(13) Dri, C.; Peters, M. V.; Schwarz, J.; Hecht, S.; Grill, L. Spatial Periodicity in Molecular Switching. Nat. Nanotechnol. 2008, 3, 649653.

(14) Zhao, H.; Sen, S.; Udayabhaskararao, T.; Sawczyk, M.; Kucanda, K.; Manna, D.; Kundu, P. K.; Lee, J.-W.; Kral, P.; Klajn, R. Reversible Trapping and Reaction Acceleration within Dynamically SelfAssembling Nanoflasks. Nat. Nanotechnol. 2016, 11, 82-88.

(15) Aviram, A.; Ratner, M. Molecular Rectifiers. Chem. Phys. Lett. 1974, 29, 277-283.

(16) Heath, J. R.; Ratner, M. A. Molecular Electronics. Phys. Today 2003, 56, 43-49.

(17) Joachim, C.; Gimzewski, J. K.; Aviram, A. Electronics Using Hybrid-Molecular and Mono-Molecular Devices. Nature 2000, 408, 541-548. 
(18) Ho, W. Single-Molecule Chemistry. J. Chem. Phys. 2002, 117

(24), 11033.

(19) Söngen, H.; Bechstein, R.; Kühnle, A. Quantitative Atomic

Force Microscopy. J. Phys.: Condens. Matter 2017, 29, 274001.

(20) Rahe, P.; Kittelmann, M.; Neff, J. L.; Nimmrich, M.; Reichling, M.; Maass, P.; Kühnle, A. Tuning Molecular Self-Assembly on Bulk Insulator Surfaces by Anchoring of the Organic Building Blocks. Adv. Mater. 2013, 25, 3948.

(21) Safiei, A.; Henzl, J.; Morgenstern, K. Isomerization of an Azobenzene Derivative on a Thin Insulating Layer by Inelastically Tunneling Electrons. Phys. Rev. Lett. 2010, 104, 216102.

(22) Repp, J.; Meyer, G.; Stojkovic, S.; Gourdon, A.; Joachim, C. Molecules on Insulating Films: Scanning Tunneling Microscopy Imaging of Individual Molecular Orbitals. Phys. Rev. Lett. 2005, 94, 026803 .

(23) Sändig, N.; Teobaldi, G.; Zerbetto, F. Polymorphism and Isomerisation of an Azobenzene Derivative on Gold. Chem. Commun. 2011, 47, 8662-8663.

(24) Meldrum, F. C. Calcium Carbonate in Biomineralisation and Biomimetic Chemistry. Int. Mater. Rev. 2003, 48, 187.

(25) Kittelmann, M.; Rahe, P.; Nimmrich, M.; Hauke, C. M.; Gourdon, A.; Kühnle, A. On-Surface Covalent Linking of Organic Building Blocks on a Bulk Insulator. ACS Nano 2011, 5, 8420-8425.

(26) Cembran, A.; Bernardi, F.; Garavelli, M.; Gagliardi, L.; Orlandi, G. On the Mechanism of the cis-trans Isomerization in the Lowest Electronic States of Azobenzene: S0, S1, and T1. J. Am. Chem. Soc. 2004, 126, 3234-3243.

(27) Kathan, M.; Hecht, S. Photoswitchable Molecules as Key Ingredients to Drive Systems away from the Global Thermodynamic Minimum. Chem. Soc. Rev. 2017, 46, 5536-5550.

(28) Gahl, C.; Brete, D.; Leyssner, F.; Koch, M.; McNellis, E. R.; Mielke, J.; Carley, R.; Grill, L.; Reuter, K.; Tegeder, P.; Weinelt, M. Coverage- and Temperature-Controlled Isomerization of an Imine Derivative on $\mathrm{Au}(111)$. J. Am. Chem. Soc. 2013, 135, 4273-4281.

(29) Antoniewicz, P. R. Model for Electron- and Photon-Stimulated Desorption. Phys. Rev. B: Condens. Matter Mater. Phys. 1980, 21, 3811-3815.

(30) Henzl, J.; Bredow, T.; Morgenstern, K. Irreversible Isomerization of the Azobenzene Derivate Methyl Orange on $\mathrm{Au}(111)$. Chem. Phys. Lett. 2007, 435, 278-282.

(31) Hagen, S.; Leyssner, F.; Nandi, D.; Wolf, M.; Tegeder, P. Reversible Switching of Tetra-Tert-Butyl-Azobenzene on a $\mathrm{Au}(111)$ Surface Induced by Light and Thermal Activation. Chem. Phys. Lett. 2007, 444, 85-90.

(32) Bazarnik, M.; Henzl, J.; Czajka, R.; Morgenstern, K. Light Driven Reactions of Single Physisorbed Azobenzenes. Chem. Commun. 2011, 47, 7764-7766.

(33) Bronner, C.; Priewisch, B.; Rück-Braun, K.; Tegeder, P. Photoisomerization of an Azobenzene on the $\mathrm{Bi}(111)$ Surface. J. Phys. Chem. C 2013, 117, 27031-27038.

(34) Wolf, M.; Tegeder, P. Reversible Molecular Switching at a Metal Surface: A Case Study of Tetra-Tert-Butyl-Azobenzene on $\mathrm{Au}(111)$. Surf. Sci. 2009, 603, 1506-1517.

(35) Dulić, D.; Molen, S. J. v. d.; Kudernac, T.; Jonkman, H. T.; Jong, J. J. D. d.; Bowden, T. N.; Esch, J. v.; Feringa, B. L.; Wees, B. J. v. OneWay Optoelectronic Switching of Photochromic Molecules on Gold. Phys. Rev. Lett. 2003, 91, 207402.

(36) Hunter, C. A.; Anderson, H. L. What is Cooperativity? Angew. Chem., Int. Ed. 2009, 48, 7488-7499.

(37) Kumagai, T.; Hanke, F.; Gawinkowski, S.; Sharp, J.; Kotsis, K.; Waluk, J.; Persson, M.; Grill, L. Controlling Intramolecular Hydrogen Transfer by Single Atoms and Molecules. Nat. Chem. 2014, 6, 41. 